\title{
FITOPLÂNCTON E FITOPERIFÍTON DE UM RIO DE ÁGUAS PRETAS DA AMAZÔNIA PERIFÉRICA DO NORTE, BRASIL
}

\author{
APRILE, F.M. ${ }^{1,2} \&$ MERA, P.A.S. ${ }^{3}$ \\ ${ }^{1}$ Pesquisador visitante Instituto Nacional de Pesquisas da Amazônia - INPA, AM, Brasil. E-mail: \\ aprilefm@hotmail.com \\ ${ }^{2}$ Instituto Oceanográfico, Universidade de São Paulo, São Paulo, Brasil. \\ ${ }^{3}$ Instituto Nacional de Pesquisas da Amazônia - INPA, AM, Brasil. E-mail: pmera@inpa.gov.br \\ Correspondência para: F. M. Aprile Instituto Oceanográfico da USP. Rua Doralice P.Teixeira 48 CEP \\ 05417-070 SP, Brasil. E-mail: aprilefm@hotmail.com.
}

\begin{abstract}
Aprile, F.M. \& Mera, P.A.S. 2007. Phytoplankton and phytoperiphyton of a black-waters river from North Peripheral Amazon, Brazil. Braz. J. Aquat. Sci. Technol. 11(2):1-14. ISSN 1808-7035. This paper aims to study the composition of the phytoplankton and phytoperiphyton and some limnological aspects in the Urubuí River basin at North Peripheral Amazon, Brazil. The samples were collected during the flood and ebb periods of 2002 and 2003. Urubuí River $\left(01^{\circ} 59^{\prime} 38^{\prime \prime}-02^{\circ} 37^{\prime} 5^{\prime \prime S}\right.$; $\left.60^{\circ} 01^{\prime} 38^{\prime \prime}-60^{\circ} 01^{\prime} 48^{\prime \prime} \mathrm{W}\right)$ is characterized by acidic water ( $\mathrm{pH} » 4.7$ ) with dark color, a low sediment load, and high content in dissolved organic matter derived from broken down plant material and from the production of humic substances. The river is controlled by the annual inundation of the Negro River. Temperature, $\mathrm{pH}$, conductivity, oxygen, $\mathrm{COD}$, potassium, total nitrogen, nitrate, nitrite, ammonium, phosphate, and silica were measured in four stations of the river. During this survey, 147 taxa were recognized and chlorophytes (49.7\%) and chromophytes $(28.6 \%)$ were the more abundant groups. During the ebb periods the highest richness were registered with $64.7 \%$. Zygnemaphyceae $(44 \%)$, Bacillariophyceae (26\%), and Euglenophyceae (14\%) were the most representative taxonomic classes. Linear regression analyzes showed that the nitrogen forms (total $\mathrm{N}, \mathrm{N}-\mathrm{NO}_{3}{ }^{-}$and $\mathrm{N}-\mathrm{NH}_{4}{ }^{+}$) had large influence on the distribution and species richness.
\end{abstract}

Key-words: composition, taxonomic list, algae, black-water, floodplain.

\section{INTRODUÇÃO}

Dentre os processos biológicos que ocorrem em um ecossistema aquático, aqueles associados à assembléia fitoplanctônica são de fundamental importância. O fitoplâncton pode contribuir com até $87 \%$ da produção primária global de um ambiente aquático natural (Likens, 1975), além de constituir a base da cadeia alimentar aquática. O estudo da composição taxonômica do fitoplâncton no estado do Amazonas é extremamente escasso, frente a sua rica biodiversidade e a complexidade de suas imensas bacias de drenagem, representadas pelos diversos ecossistemas aquáticos existentes na região. Os primeiros trabalhos de composição do fitoplâncton em sistemas de águas pretas amazônicas são apresentados por Uherkovich (1976, 1981, 1984) e Uherkovich \& Rai (1979) na bacia do rio Negro. Destacam-se ainda os trabalhos de Förster (1963, 1964, 1969 e 1974); Mera (1995) e Uherkovich \& Schmidt (1974) na Amazônia Ocidental e Amazônia Periférica do Norte. O Estado do Amazonas apresenta uma carência de trabalhos sobre dados biológicos, reforçando a necessidade de mais estudos sobre a composição microbiológica dos sistemas fluviais.

Este trabalho teve como objetivos estudar a composição e distribuição dos organismos fitoplanctônicos e fitoperifíticos presentes na bacia do rio Urubuí, no Município de Presidente Figueiredo - Estado do Amazonas, evidenciando a influência do ciclo hidrológico e das variáveis limnológicas sobre a distribuição espaçotemporal das assembléias.

\section{MATERIAL E MÉTODOS}

Área de estudo

O rio Urubuí é um rio de águas pretas localizado próximo ao Município de Presidente Figueiredo na Amazônia Periférica do Norte, no Estado do Amazonas, e limitando-se ao sul com a Amazônia Central. O rio possui uma extensão aproximada de $27 \mathrm{~km}$ desde suas nascentes até a foz no rio Urubu. $O$ trecho de estudo está compreendido entre as coordenadas $01^{\circ} 59^{\prime} 38,7^{\prime \prime}-02^{\circ} 37^{\prime} 5^{\prime \prime} \mathrm{S}$ e $60^{\circ} 01^{\prime} 38,0^{\prime \prime}-60^{\circ} 01^{\prime} 48,9^{\prime \prime} \mathrm{W}$ (Figura 1). O sistema formado pelo rio Urubuí e tributários, compreende pequenas planícies inundáveis, com áreas de praias contendo areias brancas, e igapós com vegetação terrestre temporariamente inundada. $O$ ecossistema é regido pelo ciclo hidrológico, que tem forte influência nos processos bióticos e abióticos locais. A bacia do rio Urubuí é formada pela mistura de águas claras, na maioria das vezes originadas em nas- 
centes subterrâneas, e águas pretas oriundas dos igarapés de floresta. Como resultado, tem-se águas com coloração vinho, baixa carga sedimentar, alta transparência e um alto conteúdo de material orgânico dissolvido, decorrente da produção de substâncias húmicas. Os ácidos húmicos, produzidos pela decomposição do material vegetal, são responsáveis pela forte acidez dessas águas ( $\mathrm{pH} 4,0$ a 4,6). A planície de inundação da bacia Amazônica contém centenas de lagos e paranás, muitos dos quais apresentando um hipolímnio anóxico por um período que pode compreender todo ciclo hidrológico. No trecho estudado, em particular, a peculiaridade é a presença de várias corredeiras e cachoeiras, que permitem a oxigenação de toda a coluna de água. $O$ pulso de inundação, decorrente da variação do ciclo hidrológico, causa profundas modificações no ecossistema aquático (Junk, 1984) exigindo profundas adaptações por parte da flora e fauna local.

Foram realizadas coletas em quatro pontos de amostragem na bacia do rio Urubuí (Figura 1) durante os períodos de cheia (julho-agosto) e vazante (novembro-dezembro) dos ciclos hidrológicos 2002 e 2003, totalizando 16 amostragens. Os pontos foram assim definidos: ponto A no igarapé das Lages, principal afluente do Urubuí, contendo águas ácidas provenientes da floresta; ponto B localizado no rio Urubuí acima da corredeira aproximadamente $1,8 \mathrm{~km}$; ponto $\mathrm{C}$ no trecho de corredeira, onde há diversos afloramentos de rocha basáltica e presença de troncos e outros materiais vegetais que formam barreiras à água; ponto $D$ no rio Urubuí distando abaixo da corredeira cerca de $2 \mathrm{~km}$, já próximo a sua foz, onde já se pode observar alguma atividade de banhistas e construções.

Fig. 1

\section{Procedimentos Metodológicos}

A amostragem qualitativa do fitoplâncton foi feita com arrastos horizontais com rede de abertura de malha $20 \mu \mathrm{m}$, e do fitoperifíton através de coleta manual junto a diversos substratos naturais (caules, raízes e troncos). Todo material foi estocado em frascos de polietileno e fixado em solução Transeau 1:1 (Bicudo \& Bicudo, 1970). O material coletado foi identificado e incorporado a Curadoria das coleções de algas amazônicas do Instituto Nacional de Pesquisas da Amazônia. As amostras foram identificadas em microscópio invertido Olympus 400x com contraste de fase. O corante empregado para evidenciar o amido foi o lugol, e para a bainha de mucilagem foi usada solução de azul de metileno 4\%. A identificação sistemática foi realizada por análise comparativa, e por análise de variabilidade morfológica e morfométrica dos espécimes coletados. Os sistemas de classificação adotados foram: Round (1971), Simonsen (1979), Anagnostidis \& Komárek (1988) e Van Den Hoeck (1994). Foram ela- boradas pranchas contendo fotografias em escala das espécies identificadas.

A coleta e preservação das amostras de água foram realizadas segundo recomendações de Goltermann et al. (1978). As amostras foram obtidas com garrafa de Van-Dorn e subamostras foram conservadas a $-4^{\circ} \mathrm{C}$. No campo foram aferidas temperatura da água $\left({ }^{\circ} \mathrm{C}\right), \mathrm{pH}$, condutividade $\left(\mu \mathrm{S} . \mathrm{cm}^{-1}\right)$ e oxigênio dissolvido (mg. $\left.\mathrm{L}^{-1}\right)$ através de leitura direta por potenciômetros WTW. Em laboratório foram determinados demanda química de oxigênio (DQO mg.L-1), utilizando o $\mathrm{KMnO}_{4}$ como agente oxidante; potássio ( $\mathrm{K}$ $\left.\mathrm{mg} \cdot \mathrm{L}^{-1}\right)$, por espectrofotometria de absorção atômica; $\mathrm{N}-\mathrm{NH}_{4}{ }^{+}\left(\mathrm{mg} \cdot \mathrm{L}^{-1}\right)$, a partir da reação com fenol e hipoclorito em meio alcalino e catalisada com nitroprussiato de sódio; nitrito $\left(\mathrm{N}-\mathrm{NO}_{2}{ }^{-} \mathrm{mg} \cdot \mathrm{L}^{-1}\right)$, determinado em presença de sulfanilamida preparada em meio ácido e n-naftil etilenodiamina diclórico em meio alcoólico; nitrato $(\mathrm{N}$ $\mathrm{NO}_{3}^{-} \mathrm{mg} \cdot \mathrm{L}^{-1}$ ), determinado em coluna de cádmio no qual o N-NO ${ }_{3}^{-}$é quantitativamente reduzido a $\mathrm{N}-\mathrm{NO}_{2}^{-}$em amostras filtradas e tamponadas com cloreto de amô-

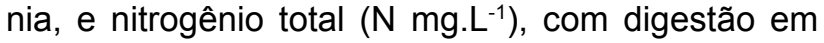
autoclave por 30' com solução de oxidação de persulfato de potássio e ácido bórico (Golterman et al., 1978; APHA, 1992). Os teores de Silicato (Si mg. $\mathrm{L}^{-1}$ ) foram analisados pelo método do molibidato de sódio $\left[\mathrm{Na}_{2} \mathrm{MoO}_{4}\right]$, seguido de acidificação $\left[\mathrm{H}_{2} \mathrm{SO}_{4}\right]$ e adição

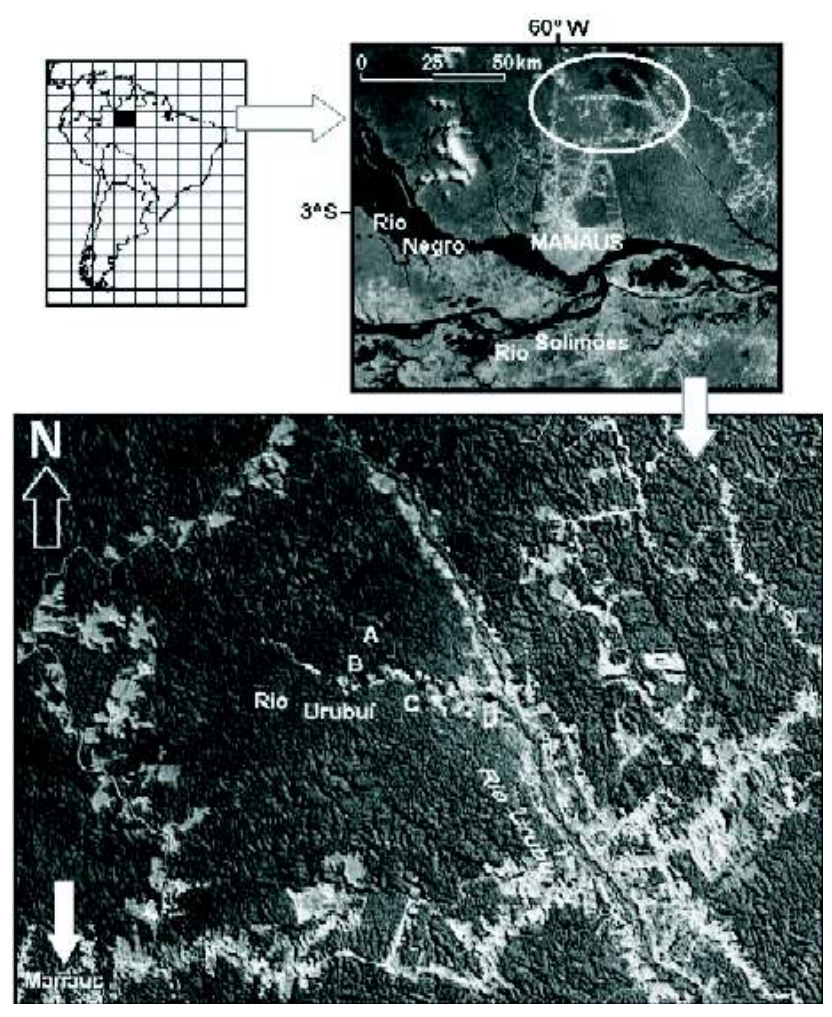

Figura 1 - Localização dos pontos de amostragem na bacia do rio Urubuí, Amazônia Periférica do Norte - Brasil. 
de $\left[\mathrm{SnCl}_{2}\right]$ para o desenvolvimento da cor azul com leitura espectrofotométrica a $810 \mathrm{~nm}$ (Wetzel \& Likens, 2000). Fosfato total $\left(\mathrm{P}_{-} \mathrm{PO}_{4}{ }^{3-} \mathrm{mg} \cdot \mathrm{L}^{-1}\right)$ foi determinado pelo método do ácido ascórbico, com extração ácida $\left(\mathrm{H}_{2} \mathrm{SO}_{4}\right.$ $+\mathrm{HCl}$ ), seguido de leitura em espectrofotômetro a 725 $\mathrm{nm}$ (Aprile \& Bianchini Jr, 2003). As inter-relações envolvendo as componentes nutricionais e a análise qualitativa do fitoplâncton e fitoperifíton foram estabelecidas através da aplicação da análise de regressão linear.

\section{RESULTADOS E DISCUSSÃO}

Com relação à composição, foram identificados 147 táxons e 136 espécies, distribuídos em seis divisões taxonômicas (Tabela 1). As Chlorophyta foram as mais representativas com $49,7 \%$ dos táxons identificados. O segundo grupo mais representativo foi Chromophyta com 28,6\%, seguido pela Euglenophyta com 14,3\%, Cyanophyta com 3,4\%, Rhodophyta com $2,7 \%$ e Phyrrophyta com 1,4\% (Figura 2A). Do total de táxons identificados, a Classe Zygnemaphyceae representou 44,2\%, Bacillariophyceae 25,9\%, Euglenophyceae 14,3\%, Chlorophyceae 4,8\%, Cyanophyceae 3,4\%, Rhodophyceae 2,7\%, Chrysophyceae 2\%, Dinophyceae $1,4 \%$ e, Oedogoniophyceae e Xanthophyceae representaram $0,7 \%$ dos táxons (Figura 2B). As ordens com maior riqueza de espécies foram: Desmidiales com 39,5\%; Euglenales 14,3\%; Ordem Eunotiales com 12,9\% e Ordem Naviculales com $9,5 \%$. Nas três Classes com maior representatividade de táxons, os gêneros mais encontrados foram: nas Zygnemaphyceae, Closterium (9,5\%), Cosmarium (7,5\%), Staurastrum $(6,1 \%)$ e Actinotaenium (4,8\%); nas Bacillariophyceae, Eunotia com $10,9 \%$ do total dos táxons, e nas Euglenophyceae, Trachelomonas com 5,4\% dos táxons estudados.

Na Tabela 1 é apresentado o resultado da análise qualitativa, bem como está demonstrada a representatividade espaço-temporal das espécies fitoplanctônicas, sendo estes resultados sumarizados na Figura 3. A maior representatividade de espécies foi observada durante a fase de vazante (Figura 3), sendo $64,7 \%$ dos táxons encontrados nesse período. A riqueza mais alta de gêneros foi obtida nos pontos $A$ e $B$ (ver Figura 1), localizados mais ao interior da bacia fluvial, em uma área bem preservada e rodeada de igarapés de floresta. É provável que nesse trecho de rio tenha havido influência direta da matéria orgânica e dos nutrientes de origem alóctone. Nestes pontos, a representatividade de espécies encontradas na vazante foi de $60,1 \%$ e $63,5 \%$, respectivamente. Nos pontos $\mathrm{C}$ e $\mathrm{D}$, onde há influência direta da velocidade de corrente do rio, os resultados mostraram uma menor representatividade de espécies, apesar disso, a dife- rença entre as fases de vazante e cheia foi igualmente marcante (Figura 3). O igarapé das Lages (ponto A) contribuiu com $83,8 \%$ de táxons para o sistema estudado, confirmando desta maneira que a bacia do rio Urubuí, como bacia de drenagem maior, atua como receptora dos tributários menores. Os igarapés de floresta da região Amazônica são os principais responsáveis pela alta diversidade biológica dos ecossistemas aquáticos. Além disso, eles exercem um papel fundamental no fornecimento de nutrientes oriundos dos processos de decomposição e lixiviação da floresta, que anualmente são redistribuídos pela planície amazônica por ação do pulso de inundação.

As pranchas de 1 a 10 apresentam as espécies fitoplanctônicas e fitoperifíticas encontradas na bacia do rio Urubuí com a respectiva lista taxonômica. As espécies filamentosas e unicelulares mais comuns do fitoperifíton, citando Spirogyra ellipsospora, $S$. rhizobrachialis, Tribonema sp, Eunotia arcus, Tabellaria fenestrata, T. flocculosa e Desmogonium femuriforme, apresentadas nas Pranchas de 1 a 3, 6, 8 e 10, foram mais freqüentes. Isto se deve ao fato que as espécies filamentosas têm a capacidade de fixação no substrato rochoso, não sendo, portanto transportadas com a corrente. As espécies unicelulares são epífitas e, portanto também não foram transportadas. Existe uma coerência estrutural na arquitetura das algas perifíticas, podendo ocorrer relações específicas (Ross, 1983), como foi observado o desenvolvimento de algumas espécies de algas sobre outras.

Durante esta pesquisa foram observados 19 táxons com predominância sobre os demais, sendo a quase totalidade dos táxons inseridos nos Grupos Chlorophyta (10 táxons) e Chromophyta (7 táxons). Nas Chlorophyta as Ordens que apresentaram espécies predominantes foram: Chlorococcales com 1 táxon; Zignemathales com 4 táxons e Desmidiales com 5 táxons. Já nas Chromophyta, houve predominância das Ordens Tribonematales (1 táxon), Diatomales (2 táxons) e Eunotiales (4 táxons). Tanto as Desmidiales quanto as Diatomales são acidófilas, tendo suas ocorrências e desenvolvimentos facilitados pelo ambiente ligeiramente ácido, característico de rios de águas pretas. Nesse contexto, o gênero Closterium e as espécies Cosmarium contractum e Staurodesmus mamillatus (Prancha 7), Tabellaria fenestrata (Pranchas 1 e 2) e Tabellaria flocculosa (Prancha 1), apresentam boa ocorrência, distribuição e dominância nas águas ácidas amazônicas. A ordem Zignematales, representada pelo gênero Spirogyra e pela espécie Mougeotia robusta, apresentou táxons do fitoperifíton, que pela capacidade de se fixar ao substrato, mantém-se ao longo do ciclo hidrológico apresentando comportamento predominante em alguns pontos de amostragem. 
Tabela 1 - Análise qualitativa do fitoplâncton e fitoperifíton do rio Urubuí demonstrando a representatividade espaço-temporal.

\begin{tabular}{|c|c|c|c|}
\hline $\begin{array}{c}\text { Pontos de amostragem } \\
\text { Período }\end{array}$ & $\begin{array}{l}\text { A B C D } \\
\text { veveveve }\end{array}$ & $\begin{array}{c}\text { Pontos de amostragem } \\
\text { Período }\end{array}$ & $\begin{array}{l}\text { A B C D } \\
\text { veveveve }\end{array}$ \\
\hline CHLOROPHYTA & & C. lagoense var. amoebum & $-x-\cdots$ \\
\hline Classe Chlorophyceae & & C. pyramidatum var. stephani & $-x----$ \\
\hline Ordem Chlorococcales & & C. pseudopyramidatum f. minus & $x x-x-x x x$ \\
\hline Ankistrodesmus falcatus & $\mathrm{xx}-\ldots$ & C. pseudopyramidatum var. borgei & $x--x-x--$ \\
\hline Coelastrum proboscidium & $\mathrm{xxxxxxx}$ & C. pseudobroomei & $-\ldots-\ldots$ \\
\hline Coenocystis piscinalis & $\mathrm{d} \times \mathrm{x} \times \mathrm{x} \times \mathrm{x}$ & C. ralfsii & $----x$ \\
\hline Oocystis lacustris & $-x---x$ & Euastrum sp. & $-x x--x-$ \\
\hline Scenedesmus quadricauda & $-x-----$ & Euastrum sinuosum & $-x-x-x--$ \\
\hline Tetraëdron gracile & $x x-x-x-x$ & $\begin{array}{l}\text { E. sinuosum var. subgangense } f \text {. } \\
\text { ellipticum }\end{array}$ & $-\mathrm{x} \times \mathrm{x}----$ \\
\hline Ordem Trentepolhiales & & E. sinuosum var. subgangense & $x--x-x--$ \\
\hline Trentepolhia sp. & $-x--x-x-$ & Netrium digitus & $\mathrm{x} \times \mathrm{x} \times \mathrm{x} \times \mathrm{x}$ \\
\hline Classe Oedogoniophyceae & & N. digitus var. lamellosum & 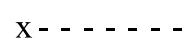 \\
\hline Ordem Oedogoniales & & N. oblongum var. cilyndricum & $x-\ldots-\ldots$ \\
\hline Oedogonium sp. & $--\mathrm{xxx}-\mathrm{x}-$ & Penium polymorphum & $\mathrm{x}-\ldots-\ldots$ \\
\hline Classe Zygnemaphyceae & & Pleurotaenium minutum & $x--x-x x x$ \\
\hline Ordem Desmidiales & & P. minutum var. attenuatum & $\mathrm{xxxx}-\mathrm{xx}-$ \\
\hline Actinotaenium cucurbita & $-x---x x$ & P. minutum var. bourrellyi & $x x-x-x-x$ \\
\hline A. cucurbita & $x x x x-x-x$ & P. trabecula & $\mathrm{xx}-\mathrm{x}---$ \\
\hline A. globosum & $x x-x-x--$ & Spondylosium desmidiiforme & $-x---x$ \\
\hline A. palangula & $\mathrm{xx}-\ldots$ & Staurodesmus mamillatus & $d d \times x \times x \times x$ \\
\hline A. peniomorphum var. latius & $\mathrm{xx}-\cdots \mathrm{xx}$ & Staurastrum fittkaui & $-\ldots x-$ \\
\hline A. rufescens & $\operatorname{xxxxxxx}$ & S. forticulatum var. minus & $-x-x-x--$ \\
\hline A. wollei & $-x-x-x-x$ & S. hagmannii & $-x-\ldots-$ \\
\hline Bambusina brebissonii & $x x-x-x-x$ & S. octangulare & $-x-x-x--$ \\
\hline Closterium abruptum & $\mathrm{x}-\ldots-\ldots$ & S. orbiculare & $-x-x-x x x$ \\
\hline C. acutum & $\mathrm{xxxx}-\mathrm{x}--$ & S. orbiculare var. ralfsii & $-x----x$ \\
\hline C. cynthia & $\mathrm{x}-\ldots-\ldots$ & S. punctulatum & $-x----$ \\
\hline C. dianae & $\mathrm{xx}-\cdots$ & S. quadrangulare var. longispinum & $-x-x--x$ \\
\hline C. kuetzingii & $-x-x x x--$ & S. teliferum var. longispinum & $-x---x$ \\
\hline C. libellula & $x x-x-x--$ & Tetmemorus laevis & $-x-x-x--$ \\
\hline C. lineatum & $\mathrm{xxxxxxx}$ & Ordem Zignemathales & \\
\hline C. malmei & $\mathrm{x}-\ldots-\ldots$ & Mougeotia robusta & $x d x-x x-x$ \\
\hline C. navicula & $x d x x x x x x$ & Spirogyra ellipsospora & $x \times x \times x \times d d$ \\
\hline C. parvulum & $x x x d-d-x$ & S. rhizobrachialis & $-x_{x x d d d}$ \\
\hline C. ralfsii & $x \times x d x x x$ & S. crassa & $-\cdots x-x$ \\
\hline C. regulare var. regulare & $\mathrm{x}--\mathrm{x}---\mathrm{x}$ & S. subsalsa & $-\ldots-x_{-}^{-}$ \\
\hline C. regulare var. regulare $f$. minus & $x--x-x-x$ & S. aequinoctialis & $-x--d-x$ \\
\hline C. setaceum & $-x-x-x--$ & Ordem Ulotrichales & \\
\hline Cilyndrocystis crassa & $-x----$ & Geminella sp. & $x-\cdots--$ \\
\hline Cosmarium bipunctatum & $x-x x-x--$ & EUGLENOPHYTA & \\
\hline C. contractum & $d d x x \times x \times x$ & Classe Euglenophyceae & \\
\hline C. depressum var. achondrum & $\mathrm{xxxxx---}$ & Ordem Euglenales & \\
\hline C. granatum & $---x-x--$ & Astasia curvata & $-x----$ \\
\hline C. elegantissimum & $-\ldots-\ldots$ & Euglena acus & $x x-x-x x-$ \\
\hline
\end{tabular}

$v$ = vazante; $c$ = cheia; $x=$ presença; - = ausência; $d$ = dominância 


\begin{tabular}{|c|c|c|c|}
\hline $\begin{array}{c}\text { Pontos de amostragem } \\
\text { Período }\end{array}$ & $\begin{array}{l}\text { A B C D } \\
\text { veveveve }\end{array}$ & $\begin{array}{c}\text { Pontos de amostragem } \\
\text { Período }\end{array}$ & $\begin{array}{l}\text { A B C D } \\
\text { vevevevC }\end{array}$ \\
\hline E. grisoli & $-x----$ & E. arcus & $\overline{x x x d x x d x}$ \\
\hline E. oxyuris & $-x--\ldots$ & E. asterionelloides & $\mathrm{d} \times \mathrm{x} \times \mathrm{x} \times \mathrm{x}$ \\
\hline E. spirogyra & $-\mathrm{x}-----$ & E. bilunaris & $x x x-x-x-$ \\
\hline Lepocinclis fusiformis & $-x---x--$ & E. bilunaris var. mucophila & $\mathrm{x} \times \mathrm{x} \times \mathrm{x}-\mathrm{x}-$ \\
\hline L. salina & $-x-x-x x x$ & E. conversa & $--x-x x-$ \\
\hline L. ovum & $-x---x$ & E. faba & $\mathrm{x} \times \mathrm{x} \times \mathrm{x} \times \mathrm{x}$ \\
\hline Phacus anomalus & $x x-x-x-x$ & E. flexuosa & $x d-x--x x$ \\
\hline P. longicauda & $-x-x-x-x$ & E. grunowii & $---x-x x-$ \\
\hline$P$ gigas & $-\mathrm{x}----$ & E. intermedia & $\mathrm{xx}--\mathrm{x}-\mathrm{xx}$ \\
\hline P. quinque-marginatus & $-x-x---$ & E. muelleri & $--x x--x$ \\
\hline P. suecicus & $-x----$ & E. parallela & $--x-x--$ \\
\hline Trachelomonas armata f. inevolula & $x-\ldots-\ldots$ & E. pectinalis & $\mathrm{x} \times \mathrm{x} \times \mathrm{x} \times \mathrm{x}$ \\
\hline T. australica var. rectangularis & $-x-x-x-x$ & E. sierra & $-x-x-x-x$ \\
\hline T. cylindrica var. decollata & $-x-x-x--$ & E. triodon var. genuina & $--\operatorname{xxxxx}$ \\
\hline T. hispida var. duplex & $-x--\ldots$ & E. zygodon & $-x-x x-x-$ \\
\hline T. oblonga & $-x--\ldots$ & Ordem Naviculales & \\
\hline T. superba & $\mathrm{x}-\ldots-\ldots$ & Frustulia rhomboides & $\mathrm{x} \times \mathrm{x} \times \mathrm{xxx}$ \\
\hline T. volvocina & $-x-x-x-x$ & F. rhomboides var. crassinervia & $\mathrm{x} \times \mathrm{x} \times \mathrm{xxx}$ \\
\hline T. volvocina var. derephora & $-x----x$ & $\begin{array}{l}\text { Gomphonema archaevibrio } f \text {. } \\
\text { archaevibrio }\end{array}$ & $-x x---x$ \\
\hline PHYRRHOPHYTA & & G. subtile & $-x-x---$ \\
\hline Classe Dinophyceae & & Gyrosigma sp. & $-x----$ \\
\hline Ordem Peridiniales & & Nitzschia reversa & $-\ldots x-\ldots$ \\
\hline Gynnodinium sp. & $-\mathrm{xxxxx-}-$ & N. acicularis & $\mathrm{x}-\cdots-{ }^{-\cdots}$ \\
\hline Peridinium inconspicuum & $\operatorname{xxxxxxx}$ & Pinnularia braunii & $-\ldots-2 x-$ \\
\hline CHROMOPHYTA & & P. gibba & $---x---$ \\
\hline Classe Xanthophyceae & & P. hemiptera & $-x x x-x x x$ \\
\hline Ordem Tribonematales & & P. maior & $x-\cdots x x$ \\
\hline Tribonema sp. & $\operatorname{xxx} x d d x$ & Stenopterobia intermedia & $-x---x-$ \\
\hline Classe Chrysophyceae & & S. delicatissima & $\mathrm{x} \times \mathrm{x} \times \mathrm{xxx}$ \\
\hline Ordem Ochromonadales & & Surirella linearis var. constricta & $\mathrm{x} \times \mathrm{x} \times \mathrm{x} \times \mathrm{x}$ \\
\hline Mallomonas tonsurata & $-x----$ & CYANOPHYTA & \\
\hline M. longiseta & $\mathrm{x} \times \mathrm{x} \times \mathrm{xxx}-$ & Classe Cyanophyceae & \\
\hline Synura uvella & $--x-x-x$ & Ordem Chroococcales & \\
\hline Classe Bacillariophyceae & & Merismopedia glauca & $--x x--$ \\
\hline Ordem Coscinodiscales & & Ordem Nostocales & \\
\hline Aulacoseira pseudogranulata & $-x x-x-x x$ & Oscillatoria amphibia & $-x-\ldots-$ \\
\hline Ordem Diatomales & & O. nigra & $---x---$ \\
\hline Fragilaria sp. & $---x--$ & O. sancta & $x-\cdots x$ \\
\hline Synedra acus & $-x-x--x x$ & O. tenuis & $-x x-x-x x$ \\
\hline Tabellaria fenestrata & $d x x x x x d x$ & RHODOPHYTA & \\
\hline $\begin{array}{l}\text { T. flocculosa } \\
\text { Ordem Eunotiales }\end{array}$ & $d x \times x \times x d x$ & $\begin{array}{l}\text { Classe Rhodophyceae } \\
\text { Ordem Acrochaetiales }\end{array}$ & \\
\hline Actinella mirabilis & $\operatorname{xxxxxxx}$ & Audouinella violacea & $-x \times x d x x x$ \\
\hline A. robusta & $---x-x--$ & Ballia sp. (1) & $x-x \times x \times x x$ \\
\hline A. brasiliensis & $\mathrm{xxxxxxx}$ & Ballia sp. (2) & $--x \times x \times x x$ \\
\hline Eunotia femoriforme & $d x x x x x d x$ & Thorea sp. & $x---x---$ \\
\hline
\end{tabular}

$\mathrm{v}$ = vazante; $c$ = cheia; $x$ = presença; - = ausência; $d$ = dominância 


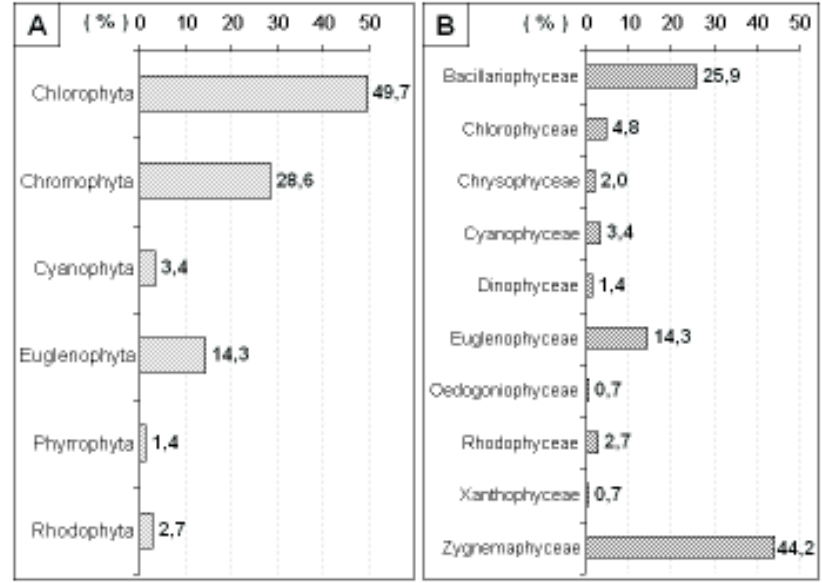

Figura 2 - A) riqueza total (\%) das divisões fitoplanctônicas e fitoperifíticas e B) riqueza total (\%) das classes de algas fitoplanctônicas e fitoperifíticas encontradas no rio Urubuí, AM Brasil durante os períodos de cheia e vazante de 2002 e 2003 $(\mathrm{N}=16)$.

A composição das espécies de microalgas depende das condições hidrológicas e limnológicas, estado trófico da água, natureza, e qualidade do substrato (Margalef, 1986; Lampert \& Sommer, 1997). Os resultados revelaram um ambiente que tende à homogeneidade quanto aos parâmetros limnológicos aferidos (Tabela 2). A temperatura da água ao longo do estudo oscilou entre 26,0 e $26,4^{\circ} \mathrm{C}$, o que é próximo da temperatura de igarapés de floresta amazônicos. Os baixos valores de $\mathrm{pH}(4,69 \pm 0,01-4,76 \pm 0,01)$ e condutividade (CE 13,3 $\pm 0,04-14,1 \pm 0,07{\mathrm{mS} . \mathrm{cm}^{-1}}^{-}$) aferidos são típicos de águas pretas com elevadas concentrações de substâncias húmicas. Os teores de $\mathrm{O}_{2}$ ficaram dentro da média para sistemas fluviais amazônicos de águas pretas, destacando-se o ponto $\mathrm{C}$, na área de influência direta das corredeiras, onde foram obtidos teores médios de $8,85 \pm 0,03$ (vazante) e $9,74 \pm 0,06$ (cheia) mg. $\mathrm{L}^{-1}$. O baixo consumo de oxigênio pelo sistema foi indicado pelos valores da DQO, que oscilaram entre 4,51 e 5,01 mg.L-1 (Tabela 2).

Diversas variáveis abióticas estão diretamente associadas à distribuição do plâncton nos ecossistemas lóticos, entre elas destacam-se: a profundidade, velocidade de corrente, transparência e concentração de nutrientes. Os elementos nitrogenados e fosfatados são potencialmente importantes, por exercer um controle na abundância do fitoplâncton em um ambiente. Segundo Goldman \& Horne (1983), a dinâmica dos ecossistemas aquáticos está diretamente associada a dois fatores abióticos: o papel da estrutura física da coluna de água e a dinâmica da utilização dos nutrientes.

As formas nitrogenadas foram os elementos que apresentaram maior tendência à variabilidade espaçotemporal. De forma geral, no aspecto espacial os re-

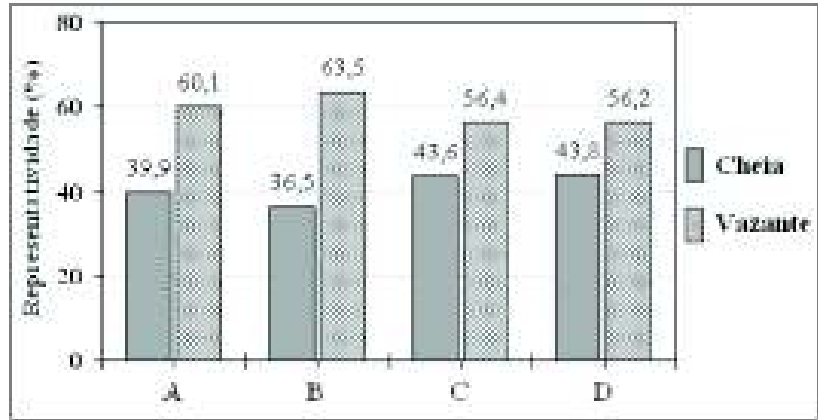

Figura 3 - Riqueza total (\%) das assembléias fitoplanctônicas e fitoperifíticas por sazonalidade (cheia e vazante) no rio Urubuí, AM, Brasil.

sultados indicaram maiores teores de $\mathrm{N}$-total, $\mathrm{N}^{-\mathrm{NO}_{3}}{ }^{-} \mathrm{e}$ $\mathrm{N}-\mathrm{NH}_{4}{ }^{+}$no igarapé das Lajes (ponto $\mathrm{A}$ ) e no rio Urubuí acima da corredeira (ponto B). Já no aspecto sazonal, os resultados mostram uma tendência a teores mais altos dessas formas nitrogenadas durante as fases de vazante. $O$ nitrogênio total variou, em média, entre $0,19 \pm 0,01$ e $0,34 \pm 0,01 \mathrm{mg} \cdot \mathrm{L}^{-1}$ nas cheias e de $0,24 \pm 0,01$ a $0,37 \pm 0,01 \mathrm{mg} \cdot \mathrm{L}^{-1}$ nas vazantes. Os teores de $\mathrm{N}-\mathrm{NO}_{3}{ }^{-}$ variaram, em termos médios, de 0,23 a $0,28 \mathrm{mg} \cdot \mathrm{L}^{-1}$ durante as cheias e de $0,25 \pm 0,01$ a $0,31 \pm 0,03 \mathrm{mg} \cdot \mathrm{L}^{-1}$


res variaram de $0,10 \pm 0,01$ para $0,29 \pm 0,01 \mathrm{mg}^{-L^{-1}}$ nas cheias e de $0,12 \pm 0,01$ a $0,32 \pm 0,01$ mg. $\mathrm{L}^{-1}$ nas vazantes (Tabela 2). Deve-se ressaltar que o ponto localizado no Urubuí à jusante das corredeiras (ponto D) apresentou concentrações de amônio ligeiramente maiores do que os pontos localizados no extremo oposto. As concentrações de nitrito apresentaram pouca variação, tanto espacialmente quanto sazonalmente, ficando próximas de $0,003 \mathrm{mg} \cdot \mathrm{L}^{-1}$. As algas podem utilizar as formas nitrogenadas $\mathrm{N}^{-} \mathrm{NO}_{3}{ }^{-}, \mathrm{N}-\mathrm{NO}_{2}{ }^{-} \mathrm{e} \mathrm{N}-\mathrm{NH}_{4}{ }^{+}$como fonte de nitrogênio para suas atividades metabólicas. $\mathrm{O}$ nitrito normalmente não é abundante em águas naturais, podendo ser até tóxico se, por algum motivo, estiver e altas concentrações. $\mathrm{O} \mathrm{N}-\mathrm{NH}_{4}{ }^{+}$é, geralmente, a forma nitrogenada preferida pelas algas, de modo que na presença de altas concentrações deste na coluna de água é assimilada preferencialmente ao nitrato. Goldman \& Horne (1983) estudando o comportamento do fitoplâncton na presença de compostos nitrogenados oriundos de esgotos domésticos, observaram um padrão de preferência do amônio ao nitrato e nitrito. $\mathrm{ON}$ $\mathrm{NH}_{4}^{+}$aparentemente é absorvido mais rapidamente pelos organismos planctônicos, enquanto que o nitrato e o nitrito precisam ser reduzidos.

O ortofosfato é reconhecidamente um dos mais importantes elementos para o plâncton, considerando o fato de este elemento poder atuar como fator limitante dos processos de produção primária (Pedersen \& Borum, 1996; Aprile \& Bianchini Jr., 2003). Espécies como as Cyanophyceae, são capazes de assimilar P- 
Tabela 2 - Valores médios \pm desvios-padrão das variáveis limnológicas analisadas na bacia do rio Urubuí (AM) durante os períodos de cheia e vazante de 2002 e 2003.

\begin{tabular}{|c|c|c|c|c|c|c|c|c|}
\hline & \multicolumn{2}{|c|}{ A } & \multicolumn{2}{|c|}{ B } & \multicolumn{2}{|c|}{ C } & \multicolumn{2}{|c|}{ D } \\
\hline & cheia & vazante & cheia & vazante & cheia & vazante & cheia & vazante \\
\hline Temp. $\left({ }^{\circ} \mathrm{C}\right)$ & $26,0 \pm 0,00$ & $26,2 \pm 0,00$ & $26,0 \pm 0,07$ & $26,2 \pm 0,07$ & $26,2 \pm 0,11$ & $26,2 \pm 0,04$ & $26,2 \pm 0,04$ & $26,4 \pm 0,25$ \\
\hline $\mathrm{pH}$ & $4,75 \pm 0,01$ & $4,73 \pm 0,00$ & $4,76 \pm 0,00$ & $4,76 \pm 0,01$ & $4,73 \pm 0,00$ & $4,72 \pm 0,00$ & $4,69 \pm 0,01$ & $4,70 \pm 0,01$ \\
\hline CE $\left(\mu \mathrm{S} . \mathrm{cm}^{-1}\right)$ & $14,0 \pm 0,01$ & $14,0 \pm 0,01$ & $14,1 \pm 0,07$ & $14,1 \pm 0,01$ & $13,6 \pm 0,08$ & $13,6 \pm 0,05$ & $13,3 \pm 0,04$ & $13,4 \pm 0,01$ \\
\hline $\mathrm{OD}\left(\mathrm{mg} \cdot \mathrm{L}^{-1}\right)$ & $6,71 \pm 0,02$ & $6,63 \pm 0,01$ & $5,51 \pm 0,19$ & $5,45 \pm 0,04$ & $9,74 \pm 0,06$ & $8,85 \pm 0,03$ & $4,91 \pm 0,06$ & $4,55 \pm 0,04$ \\
\hline $\mathrm{DQO}\left(\mathrm{mg} \cdot \mathrm{L}^{-1}\right)$ & $4,69 \pm 0,01$ & $4,51 \pm 0,09$ & $4,60 \pm 0,01$ & $4,53 \pm 0,07$ & $4,81 \pm 0,01$ & $4,73 \pm 0,06$ & $5,01 \pm 0,01$ & $4,91 \pm 0,03$ \\
\hline $\mathrm{K}\left(\mathrm{mg} \cdot \mathrm{L}^{-1}\right)$ & $1,03 \pm 0,01$ & $1,06 \pm 0,01$ & $0,96 \pm 0,01$ & $1,00 \pm 0,03$ & $0,93 \pm 0,01$ & $0,96 \pm 0,01$ & $1,03 \pm 0,01$ & $1,05 \pm 0,01$ \\
\hline $\mathrm{N}$-total $\left(\mathrm{mg} \cdot \mathrm{L}^{-1}\right)$ & $0,34 \pm 0,01$ & $0,37 \pm 0,01$ & $0,25 \pm 0,01$ & $0,29 \pm 0,01$ & $0,22 \pm 0,01$ & $0,25 \pm 0,01$ & $0,19 \pm 0,01$ & $0,24 \pm 0,01$ \\
\hline Nitrato $\left(\mathrm{mg} \cdot \mathrm{L}^{-1}\right)$ & $0,28 \pm 0,00$ & $0,31 \pm 0,03$ & $0,28 \pm 0,01$ & $0,30 \pm 0,01$ & $0,23 \pm 0,00$ & $0,26 \pm 0,01$ & $0,23 \pm 0,00$ & $0,25 \pm 0,01$ \\
\hline Nitrito $\left(m g \cdot L^{-1}\right)$ & $0,003 \pm 0,00$ & $0,003 \pm 0,00$ & $0,003 \pm 0,00$ & $0,003 \pm 0,00$ & $0,002 \pm 0,00$ & $0,003 \pm 0,00$ & $0,002 \pm 0,00$ & $0,003 \pm 0,00$ \\
\hline Amônio (mg. $\mathrm{L}^{-1}$ ) & $0,25 \pm 0,01$ & $0,26 \pm 0,01$ & $0,24 \pm 0,01$ & $0,25 \pm 0,01$ & $0,10 \pm 0,01$ & $0,12 \pm 0,01$ & $0,29 \pm 0,01$ & $0,32 \pm 0,01$ \\
\hline Fosfato $\left(\mathrm{mg} \cdot \mathrm{L}^{-1}\right)$ & $0,003 \pm 0,00$ & $0,003 \pm 0,00$ & $0,001 \pm 0,00$ & $0,002 \pm 0,00$ & $0,003 \pm 0,00$ & $0,002 \pm 0,00$ & $0,002 \pm 0,00$ & $0,003 \pm 0,00$ \\
\hline \multirow[t]{2}{*}{ Sílica $\left(\mathrm{mg} \cdot \mathrm{L}^{-1}\right)$} & $49,0 \pm 0,07$ & $49,1 \pm 0,07$ & $49,0 \pm 0,07$ & $49,1 \pm 0,07$ & $48,9 \pm 0,14$ & $49,0 \pm 0,00$ & $49,0 \pm 0,07$ & $49,1 \pm 0,07$ \\
\hline & $N=16$ & $N=16$ & $\mathrm{~N}=16$ & $\mathrm{~N}=16$ & $\mathrm{~N}=16$ & $\mathrm{~N}=16$ & $\mathrm{~N}=16$ & $\mathrm{~N}=16$ \\
\hline
\end{tabular}

$\mathrm{PO}_{4}{ }^{3-}$ além das suas necessidades, estocando-o sob a forma de grânulos de polifosfato no seu no seu citoplasma (Araújo et al., 2000). Esse processo é conhecido como "absorção de luxúria". O ortofosfato, fração inorgânica do P-total, pode ser incorporado rapidamente tanto pelo fitoplâncton (Araújo et al., 2000) como pelas macrófitas aquáticas (Aprile \& Bianchini, 2003). $\mathrm{Na}$ região do rio Urubuí, as concentrações de $\mathrm{P}-\mathrm{PO}_{4}{ }^{3-}$ variaram, em média, de 0,001 e 0,003 mg. $\mathrm{L}^{-1}$ nos períodos de cheia e entre 0,002 a $0,003 \mathrm{mg}^{-\mathrm{L}^{-1}}$ nas fases de vazante. Não foi observado para o $\mathrm{P}_{-} \mathrm{PO}_{4}{ }^{3-}$ uma clara tendência à variabilidade espaço-temporal durante o período de estudo. Durante os ciclos hidrológicos de 2002 e 2003 observou-se que a condutividade e a sílica mostraram ser parâmetros com comportamento conservativo dentro do sistema fluvial em questão. Os teores de sílica variaram, em média, de 48,9 $\pm 0,14$ a $49,0 \pm 0,07 \mathrm{mg} \cdot \mathrm{L}^{-1}$ durante as cheias, e de $49,0 \mathrm{a}$ $49,1 \pm 0,07 \mathrm{mg} . \mathrm{L}^{-1}$ durante os períodos de vazante (Tabela 2). De maneira geral, as variáveis limnológicas analisadas estão de acordo com os resultados obtidos para rios e igarapés de floresta da Amazônia Central e Periférica descritas por: Fittkau (1974); Furch (1984, 1987); Raí \& Hill (1981) e Darwich et al. (2005), onde essas características estão estreitamente ligadas com as propriedades geológicas e geoquímicas locais.

O estudo de assembléias fitoplanctônicas é fundamentalmente importante quando se procura encontrar um padrão de influência sazonal ou de interferência antrópica. Segundo Araújo et al. (2000) a sucessão fitoplanctônica pode variar segundo uma determinada interferência, em decorrência do fluxo de nutrientes de origem alóctone. Interações entre os parâmetros bióticos e abióticos podem determinar flutuações na produtividade do fitoplâncton, sendo que, a seqüência hierárquica destas interações é de difícil sistematização (Reynolds, 1984). Em se tratando de estudos sazonais, à medida que for possível associar as informações a respeito da assembléia fitoplanctônica com dados sobre os aspectos nutricionais, melhor será o entendimento dos complexos mecanismos de produtividade existentes nos ecossistemas aquáticos. A Ordem Desmidiales, representada por $39,5 \%$ dos táxons identificados, é uma constante para a maioria de sistemas aquáticos amazônicos que apresentam condições limnológicas similares as da bacia do Urubuí (Förster, $1963,1969)$. Os $25,9 \%$ dos táxons das Bacillariophyceae, com predominância do gênero Eunotia, também representam um padrão dos ambientes oligotróficos de águas claras ou pretas nas microbacias de drenagem constituídas pelos igarapés de floresta (Mera, 1995). A Ausência do caráter dominante das Cyanophyceae e Euglenophyceae, utilizadas como indicadores de ambientes eutrofizados, e os baixos teores de condutividade e nutrientes inorgânicos no sistema, refletem diretamente na estabilidade da qualidade da água da bacia do Urubuí. Uma vez que até o presente momento a bacia não sofreu nenhuma ação antrópica de grande magnitude, é provável que não tenha havido alterações que comprometam o equilíbrio da dinâmica e estrutura das populações fitoplanctônicas e fitoperifíticas.

Apesar de haver uma tendência à uniformidade química na bacia do rio Urubuí, como já foi discutido anteriormente, os resultados da análise de regressão linear (Figura 4) confirmaram haver uma influência direta das formas nitrogenadas sobre a riqueza dos organismos fitoplanctônicos e fitoperifíticos. O nitrogênio 
total apresentou correlação positiva significativa com as assembléias fitoplanctônica e fitoperifítica dos grupos Chlorophyta $\left(R^{2}=0,9303\right.$ e $\left.p<0,0001\right)$ e Euglenophyta $\left(R^{2}=0,7287\right.$ e $p<0,0001$; Figura 4a). Também foram observadas correlações significativas entre o N-NO ${ }_{3}^{-}$e a Divisão Chlorophyta $\left(\mathrm{R}^{2}=0,6428\right.$ e $p=0,0002$; Figura 4b), e entre o N-NH${ }_{4}^{+}$e a Divisão Chromophyta $\left(\mathrm{R}^{2}=0,7994\right.$ e $p<0,0001$; Figura 4c). Provavelmente, estas formas nitrogenadas estão sendo preferencialmente assimiladas pelas algas e incorporadas em seus processos biológicos, o que explica tal proporcionalidade. O fato de nem todos os grupos de algas terem apresentado correlação significativa com as formas nitrogenadas, pode estar associado à maior tendência a variabilidade espaço-temporal que estes elementos químicos apresentaram. Não foram observadas correlações significativas entre o $\mathrm{P}_{-} \mathrm{PO}_{4}{ }^{3-} \mathrm{e}$ as assembléias (Figura $4 \mathrm{~d}$ ), sugerindo que o ortofosfato não está agindo como fator limitante à riqueza fitoplanctônica e fitoperifítica nas concentrações apresentadas na água. A melhor correlação foi obtida entre o $\mathrm{P}_{-} \mathrm{PO}_{4}{ }^{3-}$ e a Divisão Euglenophyta $\left(\mathrm{R}^{2}=0,3627\right.$ e $p=0,0136$; Figura $4 d$ ).

\section{CONCLUSÕES}

A bacia do rio Urubuí está inserida em um sistema hídrico de predomínio de águas pretas, cujas principais características física e biológica são a baixa concentração de íons dissolvidos e a alta diversidade de organismos planctônicos. As divisões com maior representatividade de táxons na composição do rio Urubuí foram Chlorophyta $(49,7 \%)$ e Chromophyta (28,6\%). Regida pelo sistema hidrológico amazônico, a maior riqueza de táxons na bacia foi observada durante a vazante $(64,7 \%)$. Os pontos localizados no trecho de rio mais bem preservado, no interior da bacia, foram os que apresentaram maior riqueza de espécies, sendo que destes o igarapé de floresta foi distintamente o mais representativo. Foram identificados 19 táxons com predominância sobre os demais, sendo a quase totalidade destes pertencentes aos Grupos Chlorophyta (10 táxons) e Chromophyta (7 táxons). Dentre os fatores limnológicos estudados, as formas nitrogenadas $\mathrm{N}$ -

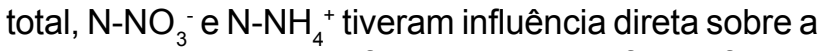
riqueza dos organismos fitoplanctônicos e fitoperifíticos.

\section{REFERÊNCIAS}

Anagnostidis, K. \& Komárek, J. 1988. Modern approach to the classification system of Cyanophytes. Arch. Hydrobiol. Suppl. 80(1-4):327-472.

APHA 1992. Standard methods for the examination of water and Wastewater. 14a ed. American Public Health Association Washington, D.C. 1193p.

Aprile, F.M. \& Bianchini Jr, I. 2003. Adequação Metodológica para Determinação de Fósforo Total em Macrófitas Aquáticas e Sedimentos. Revista Brasileira de Biociências. 8:57-64.
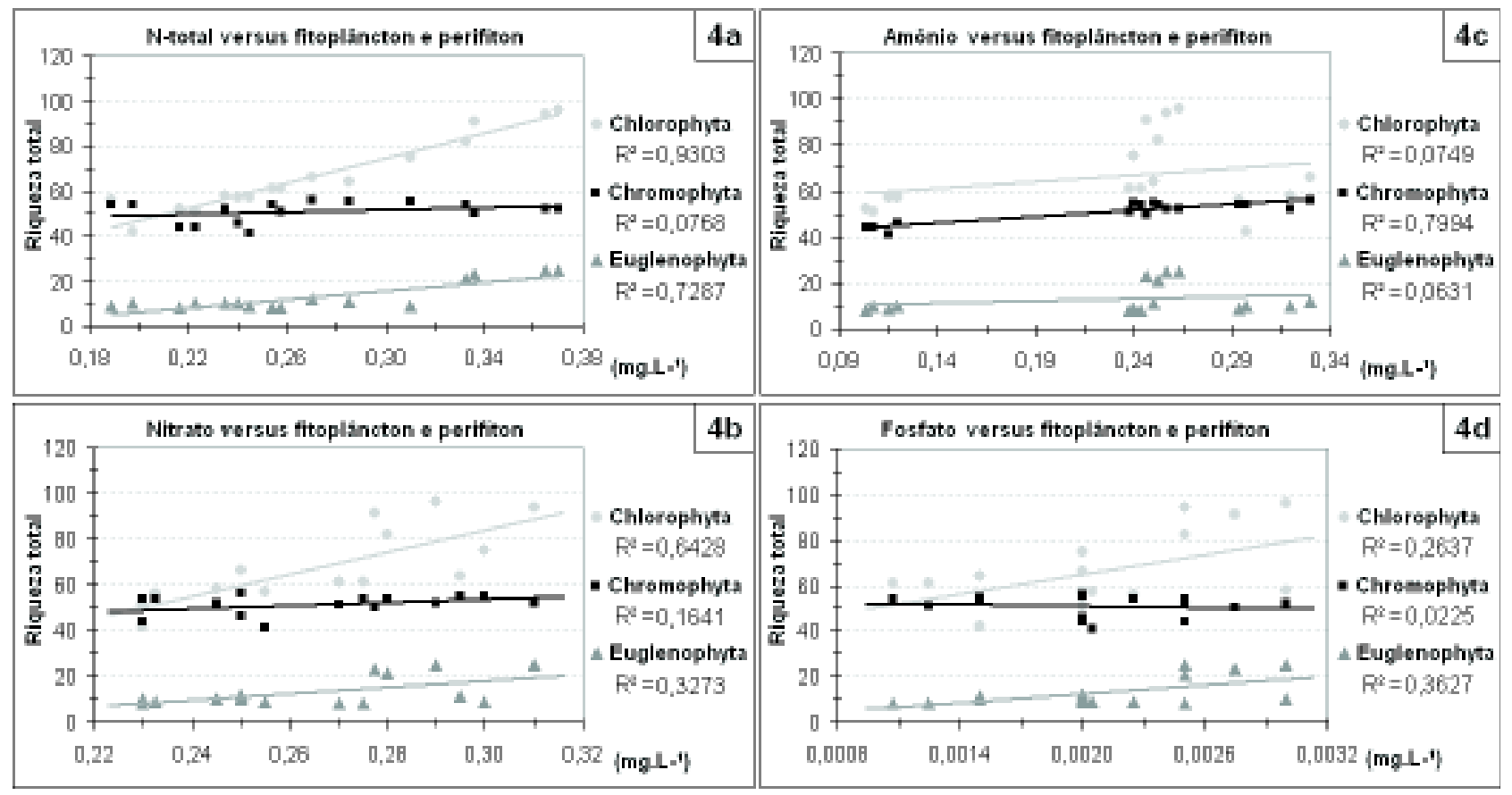

Figura 4 - Análise de regressão linear entre as formas nitrogenadas ( $\mathrm{N}$-total, $\mathrm{N}-\mathrm{NO}_{3}^{-}$e $\mathrm{N}-\mathrm{NH}_{4}^{+}$) e fosfatada $\left(\mathrm{P}-\mathrm{PO}_{4}{ }^{3-}\right)$ e as divisões fitoplanctônicas e fitoperifíticas mais representativas durante todo o período de estudo $(\mathrm{N}=16)$. 
Araújo, M.F.F.; Costa, I.A.S. \& Chellappa, N.T. 2000. Comunidade fitoplanctônica e variáveis ambientais na lagoa de Extremoz, Natal - RN, Brasil. Acta Limnol. Bras. 12:127-140.

Bicudo, C.E.M. \& Bicudo, R.M.T. 1970. Coleta e preservação de algas continentais brasileiras. São Paulo, 228p.

Darwich, A.J.; Aprile, F.M., Robertson, B.A. 2005. Variáveis limnológicas: contribuição ao estudo espaço-temporal de águas pretas amazônicas. In: Santos-Silva, E.N.; Aprile, F.M.; Scudeller, V.V.; Melo, S. (Orgs.), BioTupé: Meio Físico, Diversidade Biológica e Sociocultural do Baixo Rio Negro, Amazônia Central. INPA, Manaus, AM. 19-33pp.

Fittkau, E.J. 1974. Zur ökologischen Gliederung Amazoniens. I Die erdgeschichtliche Entwicklung Amazoniens. Amazoniana. 5(1):77-134.

Förster, K. 1963. Desmidiaceen aus Brasilien, 1: Nord Brasilien. Revue Algologique. 7(1):38-92.

Förster, K. 1964. Desmidiaceen aus Brasilien, 2 : Bahia, Goyaz, Piauhi und Nord Brasilien. Hidrobiologia. 23(3-4):321-505.

Förster, K. 1969. Amasonische Desmidiaceen, 1: Areal Santarém. Amazoniana. 2(1-2):5-23I.

Förster, K. 1974. Amazonische Desmidiaceen, 2, Areal Maués-Abacaxis. Amazoniana. 5(2):I35-242.

Furch K. 1987. Amazonian rivers: their chemistry and transport of dissolved solids through their basins. In: Mitt. Geol. Paleont. Inst. Univ. Hamburg (ed.) S.U. Sonderband, Hamburg 64, Univ. Hamburg. 285298pp.

Furch, K. 1984. Water chemistry of the Amazon basin: the distribution of chemical elements among freshwaters. In: Sioli, H. (ed.) The Amazon. Dr. W. Junk Publishers, Dordrecht. 167-200pp.

Goldman, C.R. \& Horne, A.J. 1983. Limnology. McGramHill, London. 464p.

Golterman, H.L.; Clymo, R.S. \& Ohnstad, M.A. 1978. Methods for physical and chemical analysis of fresh waters. 2nd ed. IBP Handbook n8. Blackwell Scientific Publications, Oxford. 214p.

Junk, W.J. 1984. Ecology of the várzea floodplains of Amazon white-water rivers. In: Sioli, H. (ed.) The Amazon. Dr. W. Junk Publishers, Dordrecht. 215243pp.

Lampert, W. \& Sommer, U. 1997. Limnoecology: the ecology of lakes and streams. Oxford University Press, Inc. Oxford, New York. 382p.
Likens, G.E. 1975. Primary production of inland aquatic ecosystem. In: Lieth, H. \& Whittaker, R.H. (eds.) Primary productivity of the biosphere. New York, Springer Verlag. 185-202pp.

Margalef, R. 1986. Ecologia. Ediciones Omega. Barcelona. 951p.

Mera, P.A.S. 1995. Características Ecológicas da Desmidioflórula de uma Região Hidrográfica do Sistema Trombetas, Estado do Pará, Brasil. Tese de Doutorado, INPA/FUA, Manaus, 237p.

Pedersen, M. F. \& Borum, J. 1996. Nutrient control of algal growth in estuarine waters. Nutrient limitation and the importance of nitrogen requirements and nitrogen storage among phytoplankton and species of microalgae. Mar. Ecol. Prog. Ser. 142:261-272.

Rai, H. \& Hill, G. 1981. Physical and chemical studies of lago Tupé; a Central Amazonian Black Water, Ria Lake. Int. Revue ges. Hydrobiol. 66(1):37-82.

Reynolds, C.S. 1984. Phytoplankton periodicity: the interactions of form, function and environmental variability. Freshwat. Biol. 14:111-142.

Ross, P.J. 1983. Dynamics of periphytic communities. In: Wetzel, R.G., (ed.) Periphyton of freshwater ecosystems. The Hague, Dr. W. Junk. 5-10pp.

Round, F.E. 1971. The taxonomy of the Clorophyta, 2 Br. Phycol. Jour. 6(2):235-264.

Simonsen, R. 1979. The diatom system: ideas on phylogeny. Bacillaria. 2:9-70.

Uherkovich, G. \& Rai, H. 1979. Algen aus den Rio Negro und scinen Nebenflüssen. Amazoniana. 6(4):611638.

Uherkovich, G. \& Schmidt, G.W. 1974. Phytoplanktontaxa in den Zentralamazonischen Schwemmlandensee, Lago do Castanho. Amazoniana. 5(2):243-283.

Uherkovich, G. 1976. Algen aus den Flüssen Rio Nedro und rio Tapajós. Amazoniana. 5(4):465-515.

Uherkovich, G. 1981. Algen aus cinigen Gewaessern Amazoniens. Amazoniana. 7(2):191-219.

Uherkovich, G. 1984. Phytoplankton. In: Sioli, H. (ed.) The Amazon: limnology and landscape ecology of a might tropical river and its basin. Dr. Junk Publishers, 295-310pp.

Van Den Hoek, C. 1994. Algae: an Introduction to Phycology. Cambridge University Press, 419p.

Wetzel, R.G. \& Likens, G.E. 2000. Limnolgical analysis. W. B. Saunders Co. Philadelphia. 357p. 

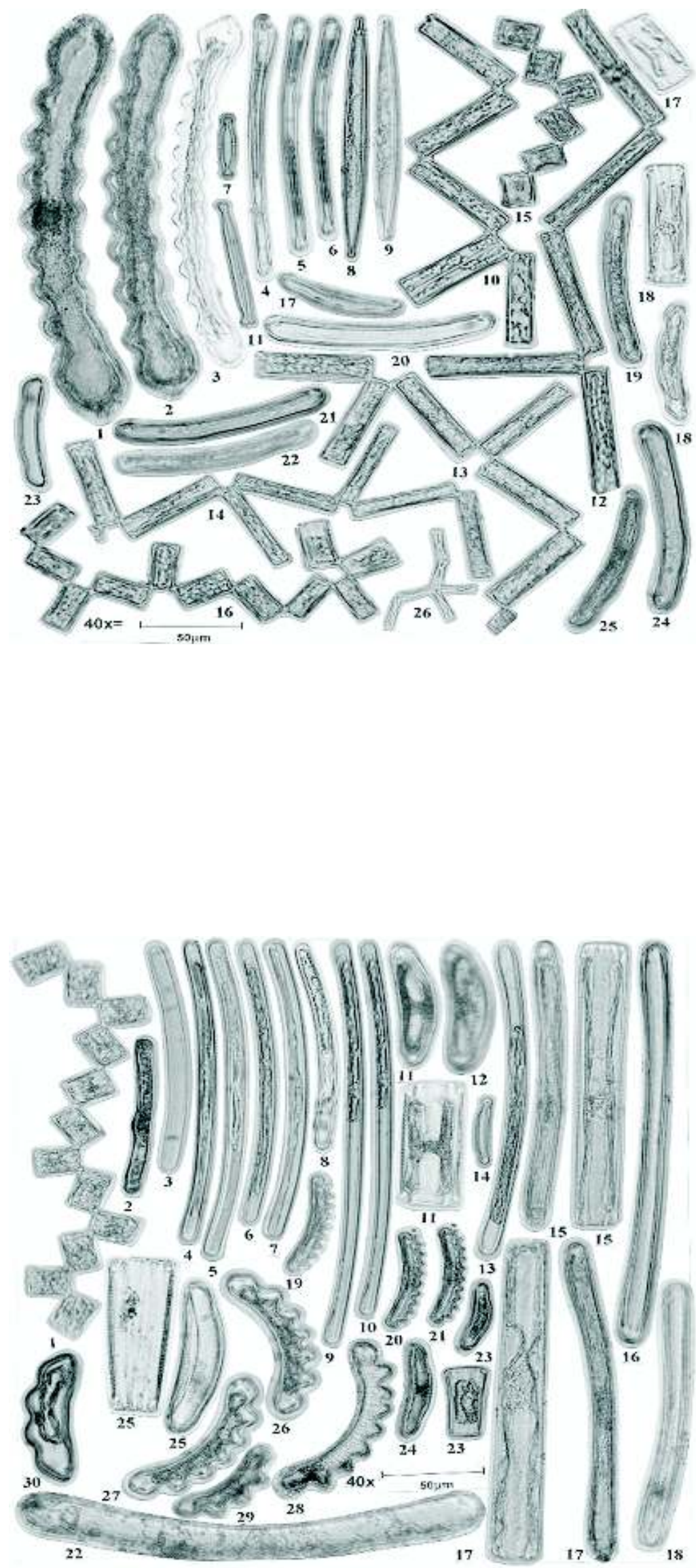

Prancha 1 - Chromophyta Fig. 1-3. Actinella mirabilis Grunow (40x); Fig. 4-6. A. robusta Hustedt (40x); Fig. 7. Fragilaria sp. (40x); Fig. 8-9. Synedra acus Kützing (40x); Fig. 10-14. Tabellaria fenestrata (Lyngbye) Kützing (40x); Fig. 15-16. T. flocculosa (Roth) Kützing (40x); Fig. 17-25. Eunotia arcus Ehrenberg (40x); Fig. 26. Eunotia asterionelloides Hustedt (40x)
Prancha 2 - Chromophyta Fig. 1. Tabellaria fenestrata (Lyngb.) Kützing (40x); Fig. 2-3. Eunotia bilunaris (Ehrenb.) Mills (40x); Fig. 4-8. E. bilunaris var. mucophila Lange-Bertalot \& Nörpel (40x); Fig. 9-10. E. conversa Hustedt (40x); Fig. 11-12. E. faba Ehrenb. (40x); Fig. 13. E. flexuosa (Brébisson) Kützing (40x); Fig. 14. E. grunowii Berg. (40x); Fig. 15-18. E. intermedia (krasske) Nörpel \& Lange-Bertalot (40x); Fig. 19-21. E. muelleri Hustedt (40x); Fig. 22. E. parallela Ehrenb. (40x); Fig. 2325. E. pectinalis (Dillwyn) Rabenhorst (40x); Fig. 26-27. E. sierra Ehreng. (40x); Fig. 28. E. sierra var. polyodon Ehrenb. (40x); Fig. 29. E. triodon var. genuina Cleve (40x); Fig. 30. E. zygodon Ehrenb. (40x). 

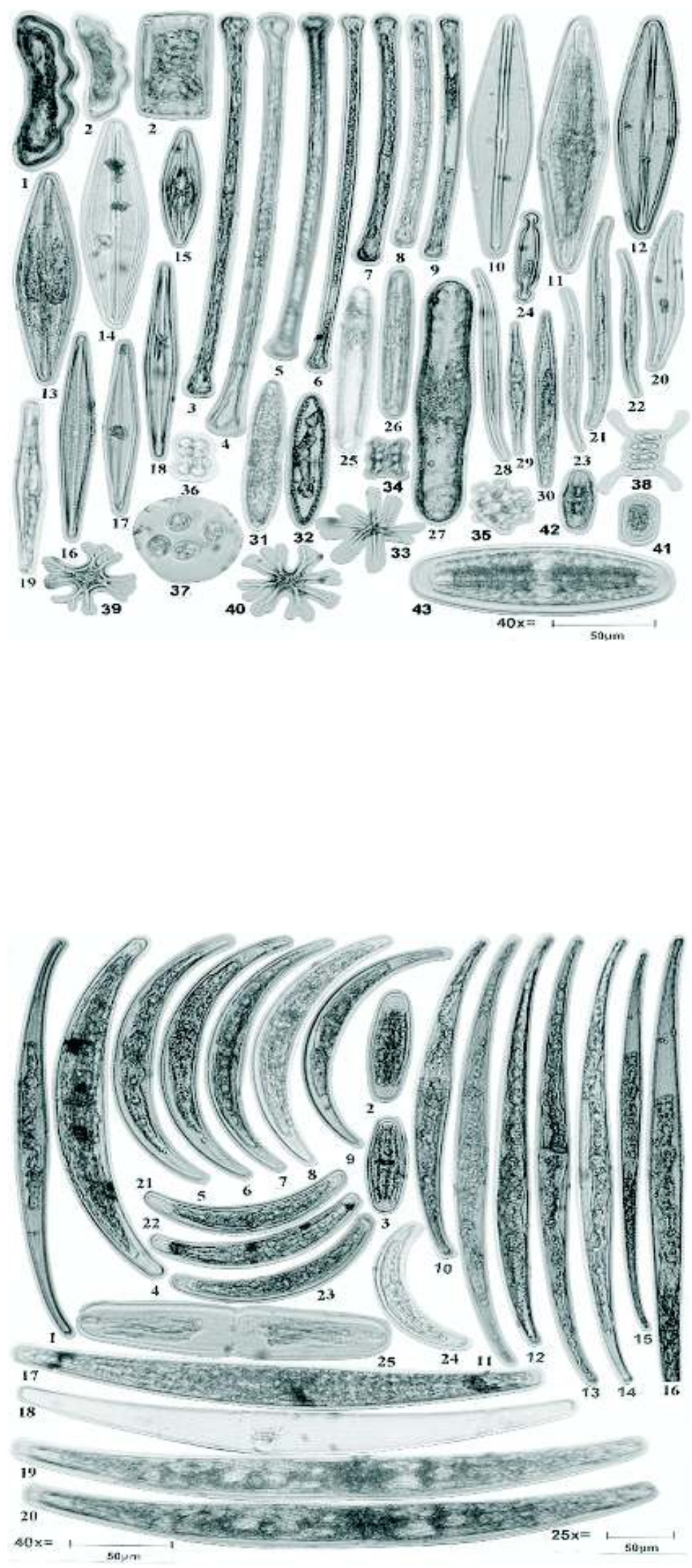

Prancha 3 - Chromophyta Fig. 1-2. Eunotia zygodon Ehrenb. (40x); Fig. 3-9. Eunotia femoriforme (Patrick) Hustedt (40x); Fig. 1014. Frustulia rhomboides (Ehrenb.) de Toni (40x); Fig. 15. F. rhomboides var. crassinervia (Brébisson) Ross (40x); Fig. 16-18. Gomphonema archaevibrio f. archaevibrio Lange-Bertalot \& Reichardt (40x); Fig. 19. G. subtile Ehrenb. (40x); Fig. 20. Gyrosigma sp. (40x); Fig. 21. Nitzschia reversa Smith (40x); Fig. 22-23. N. acicularis (Kützing) Smith (40x); Fig. 24. Pinnularia braunii (Grunow) Cleve (40x); Fig. 25. P. gibba Ehrenb. (40x); Fig. 26. $P$. hemiptera (Kützing) Cleve (40x); Fig. 27. P. maior (Kützing) Rabenhorst (40x); Fig. 28. Stenopterobia intermedia (Lewis) Van Heurck (40x); Fig. 29-30. S. delicatissima (Lewis) Van Heurck (40x); Fig. 31-32. Surirella linearis var. constricta Hustedt (40x); Chlorophyta Fig. 33. Ankistrodesmus falcatus (Corda) Ralfs (40x); Fig. 34-35. Coelastrum proboscidium Bohl (40x); Fig. 36. Coenocystis sp. (40x); Fig. 37. Oocystis lacustris Chodat (40x); Fig. 38. Scenedesmus quadricauda (Turpin) Brébisson (40x); Fig. 39-40. Tetraedron gracile (Reinsch) Hansg. (40x); Fig. 41. Cilyndrocystis crassa De Bary (40x); Fig. 42. Netrium digitus (Ehrenb.) Itzigon \& Rotle (40x); Fig. 43. N. digitus var. lamellosum (Bréb.) Grönblad (40x).
Prancha 4 - Chlorophyta Fig.1. Closterium kuetzingii Brébisson (40x); Fig. 2-3. C. navicula (Bréb.) Lütkemüller (40x); Fig. 4. C. malmei Borge (40x); Fig. 5-9. C. parvulum Nägeli (40x); Fig. 10-16. C. ralfsii Bréb. (40x); Fig. 17-20. C. regulare var. regulare Bréb. (40x); Fig. 2124. C. regulare var. regulare Bréb. f. minus Förster (40x); Fig. 25. Tetmemorus laevis (Kützing) Ralfs (40x). 

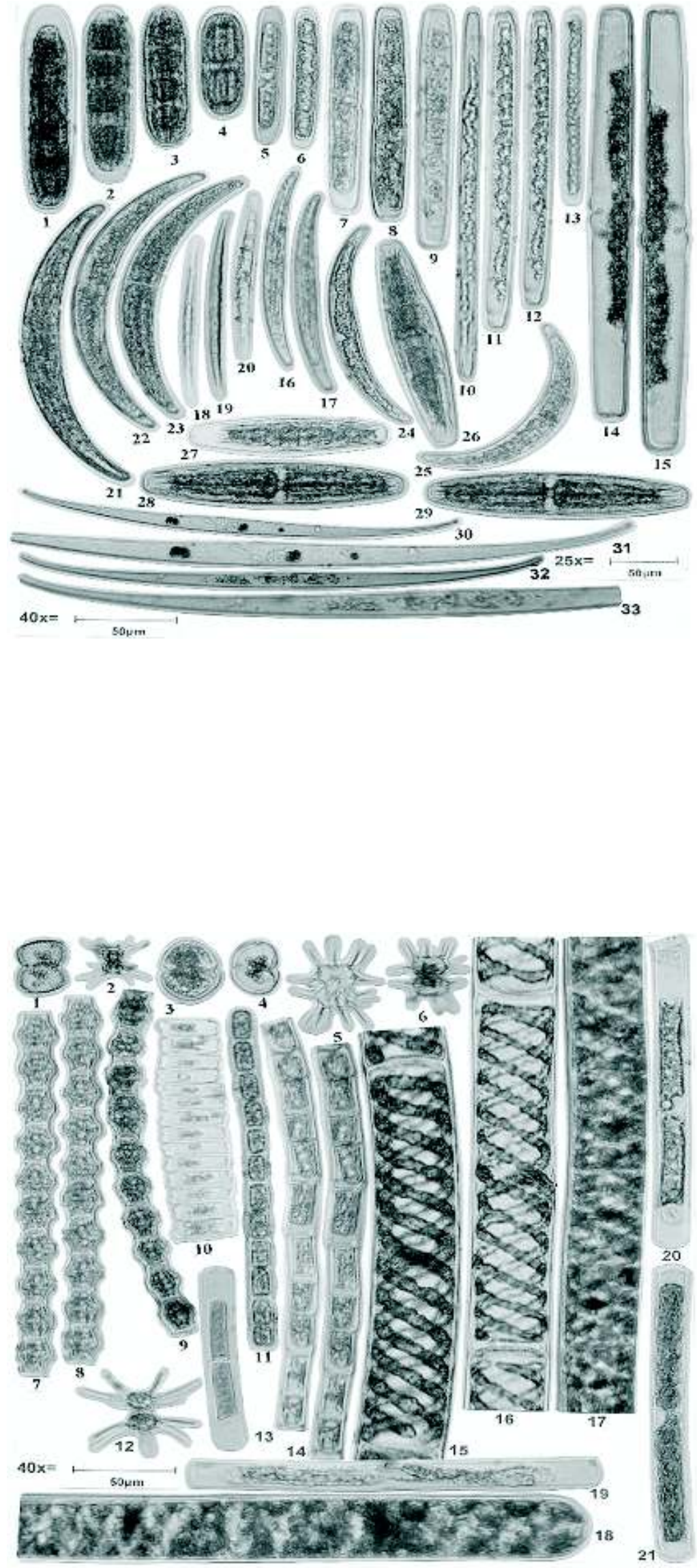

Prancha 5 - Chlorophyta Fig. 1. Netrium oblongum var. cilyndricum West \& West (40x); Fig. 2-4. Penium polymorphum Perty (40x); Fig. 5-6. Pleurotaenium minutum (Ralfs) Delponte (40x); Fig. 7-9. P. minutum var. bourrellyi Grönblad \& Scott (40x); Fig. 10-13. $P$. minutum var. attenuatum Krieger (40x); Fig. 14-15. P. trabecula (Nägeli) Ehrenb (40x); Fig. 16-17. Closterium abruptum W. West (40x); Fig. 18-20. C. acutum Brébisson (40x); Fig. 21-23. C. cynthia De Notaris (40x); Fig. 24-25. C. dianae Ehrenb. (40x); Fig. 26-29. C. libellula var.libellula f. intermedium (Roy \& Bisset) Kossinskaja (40x); Fig. 30-33. C. lineatum (Ralfs) Ehrenb. (40x).
Prancha 6 - Chlorophyta Staurastrum punctulatum (Brébisson) Ralfs (40x); Fig. 2. $S$. quadrangulare var. longispinum Börges (40x); Fig. 3. S. orbiculare (Ehr.) Ralfs (40x); Fig. 4. S. orbiculare var. ralfsii West \& West (40x); Fig. 5-6. S. teliferum var. longispinum Grönblad (40x); Fig. 7-9. Bambusina brebissonii Kützing (40x); Fig. 10. Spondylosium desmidiiforme (Borge) West (40x); Fig. 11. Geminella sp. (40x); Fig. 12. Staurastrum fittkaui Förster (40x); Fig. 13-14. Oedogonium sp. (40x); Fig. 15-16. Spirogyra ellipsospora Transeau (40x); Fig. 17-18. S. rhizobrachialis Jao (40x); Fig. 19-21. Mougeotia robusta (De Bary) Wittrock (40x). 

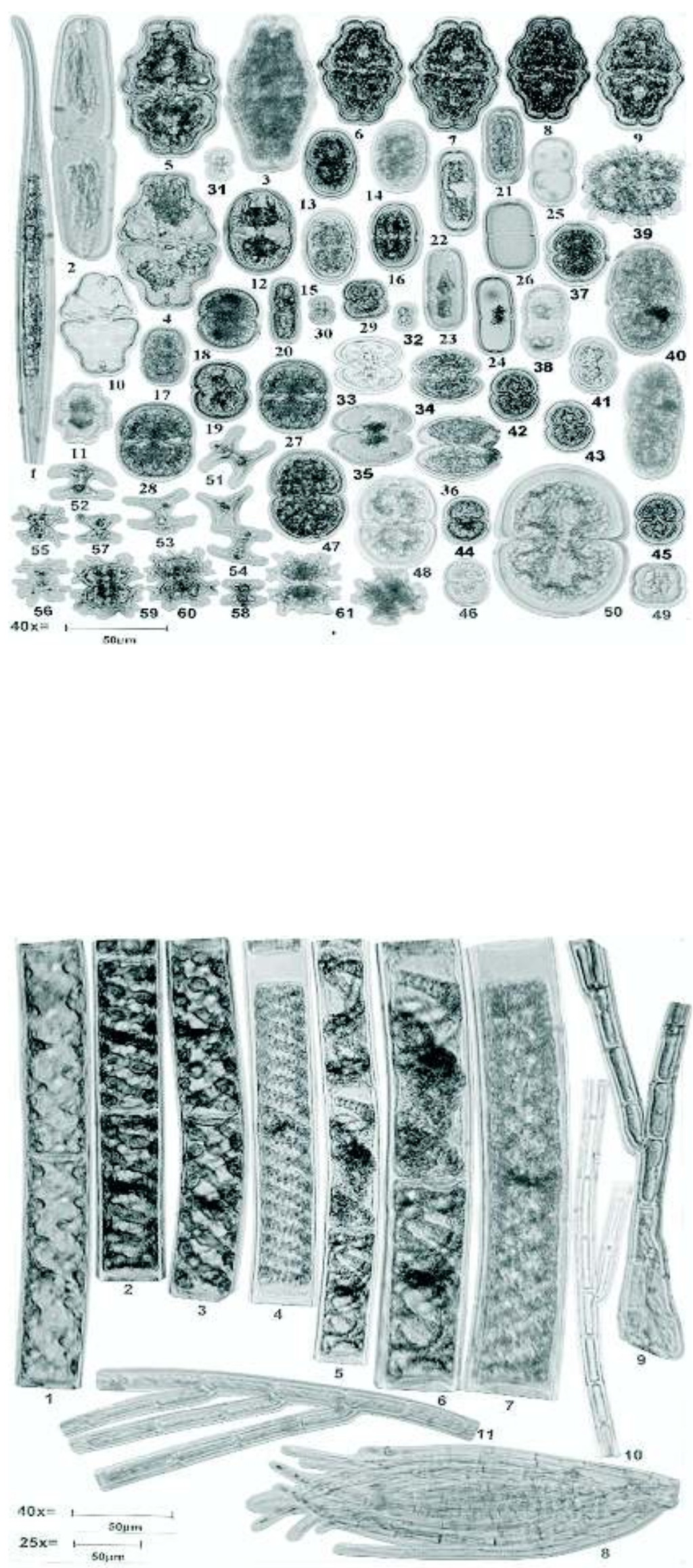

Prancha 7 - Chlorophyta Fig. 1. Closterium setaceum Ehrenberg (40x); Fig. 2. Tetmemorus laevis (Kützing) Ralfs (40x); Fig. 3. Euastrum sinuosum Lenormand (40x); Fig. 4-5. E. sinuosum var. subgangense Förster (40x); Fig. 6-10. E. sinuosum. var. subgangense f. ellipticum Förster (40x); Fig. 11. Euastrum sp. (40x); Fig. 12. Actinotaenium cucurbita (Bréb.) Teiling (40x); Fig 13-17. A. cucurbita var. cucurbita f. rotundatum (Bréb.) Teiling (40x); Fig. 18-19. A. globosum (Bulnheim) Teiling (40x); Fig. 20. A. palangula (Bréb.) Teiling (40x); Fig. 21-24. A. peniomorphum West \& West var. latius (Scott \& Prescott) (40x); Fig. 25-26. A. rufescens (Cleve) Teiling (40x); Fig.27-28. A. wollei (Grönblad) Teiling (40x); Fig. 29. Cosmarium bipunctatum Börgesen (40x); Fig. 30-32. C. contractum Kirchner (40x); Fig. 33-36. C. depressum (Nägeli) Lundell var. achondrum (Boldt) West \& West (40x); Fig. 37. C. granatum (Ralfs) Bréb. (40x); Fig. 38. C. elegantissimum Lundell (40x); Fig. 39. C. lagoense var. amoebum Förster \& Eckert (40x); Fig. 40. C. pyramidatum var. stephani (IrénéeMarie) Krieger \& Gerloff (40x); Fig. 41-46. C. pseudopyramidatum f. minus Wille (40x); Fig. 4748. C. pseudopyramidatum var. borgei Krieger \& Gerloff (40x); Fig. 49. C. pseudobroomei Wölle (40x); Fig. 50. C. ralfsii Bréb. (40x); Fig. 51-54. Staurodesmus mamillatus (Nordstedt) Teiling (40x); Fig. 55-56. Staurastrum forticulatum var. minus (Fritsh \& Rich) Grönblad \& Scott (40x); Fig. 57-58. S. hagmannii Grönblad (40x); Fig. 5961. S. octangulare Grönblad (40x).
Prancha 8 - Chlorophyta Fig. 1-3. Spirogyra rhizobrachialis Jao. (40x); Fig. 4. S. crassa Kützing (25x); Fig. 5. S. subsalsa Kützing (25x); Fig. 6-7. S. aequinoctialis G.S. West (40x); Fig. 8. Threntepohlia sp. (25x); Rhodophyta Fig. 911. Audouinella violaceae (Kützing) Hamel (40x). 

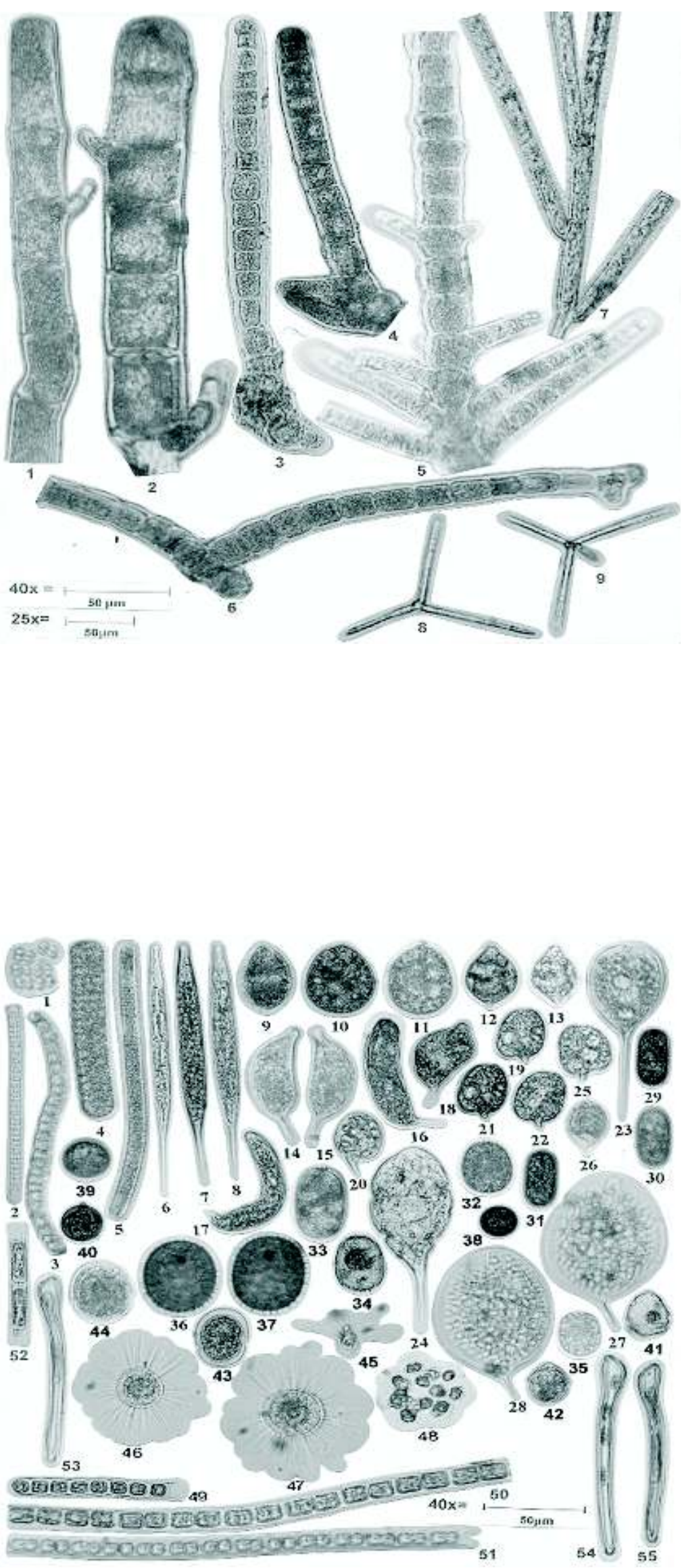

Prancha 9 - Rhodophyta Fig. 1-2. Ballia sp. (1) (40x); Fig. 3-6. Ballia sp. (2) (25x); Fig. 7. Thorea sp. (40x); Fig. 8-9. Tetrachaetum elegans Ingold (40x).
Prancha 10 - Euglenophyta Fig. 1. Merismopedia glauca (Ehrenberg) Nägeli (40x); Fig. 2. Oscillatoria amphibia Agardh (40x); Fig. 3. O. nigra Vaucher (40x); Fig. 4. O. sancta (Kützing) Gomont; Fig. 5. O. tenuis Agardh; Fig. 6-8. Euglena acus Ehrenberg (40x); Fig. 9. Lepocinclis fusiformis (Carter) Lemmermann (40x); Fig. 10-11. L. salina Fritsch (40x); Fig. 12-13. L. ovum (Ehrenb.) Minkiewicz (40x); Fig. 14-15. Astasia curvata Klebs (40x); Fig. 16. Euglena oxyuris Schmarda (40x); Fig. 17. E. grisoli Deflandre (40x); Fig. 18. E. spirogyra Ehrenb. (40x); Fig. 19-22. Phacus anomalus Fritsch \& Rich (40x); Fig. 23-24. P. longicauda (Ehrenb.) Dujardin (40X); Fig. 25. P. quinquemarginatus Jahn \& Shawhan (40x); Fig. 26. $P$. suecicus (Lemm.) Lemm. (40x); Fig. 27-28. P. gigas da Cunha (40x); Fig. 29-31. Trachelomonas australica var. rectangularis Defl. (40x); Fig. 32. T. armata f. inevolula Defl. (40x); Fig. 33. T. cylindrica var. decollata Playfair (40x); Fig. 34. T. hispida var. duplex Defl. (40x); Fig. 35. T. oblonga Lemm. (40x); Fig. 36-37. T. superba Swirenko (40x); Fig. 38-39. T. volvocina Ehrenb. (40x); Fig. 40. T. volvocina var. derephora Conrad (40x); Phyrrhophyta Fig. 41-42. Peridinium inconspicuum Lemm. (40x); Fig. 43-44. Gynnodinium sp. (40x); Chromophyta Fig. 45. Mallomonas tonsurata Krieger (40x); Fig. 46-47. M. longiseta Lemm. (40x); Fig. 48. Synura uvella Ehrenb. (40x); Fig. 49-51. Tribonema sp. (40x); Fig. 52. Aulacoseira pseudogranulata Cleve \& Simonsen (40x); Fig. 53-55. Actinella brasiliensis Grunow (40x). 